\title{
La Gerencia Pública en la perspectiva del cambio estructural
}

Pedro Medellín Torres*

\section{Resumen}

Las transformaciones empresariales parecen marcar el contenido y dirección de los cambios en el Estado y sugieren la necesidad de una óptica privada en la gerencia pública. Los cambios estatales están centrados en la descentralización -originando una novedosa territorialidad-, en la eficiencia administrativa y en la participación del ciudadano. Sin embargo, es inconveniente transferir mecánicamente modelos de gestión privada al sector público, por sus diferencias intrínsecas, por la dicotomía política-administración y por los efectos del poder burocrático. En la gerencia pública, prevalece la racionalidad política sobre la técnica, a pesar que en un escenario de cambio la acción gerencial pública pareciera vaciarse de contenido político, aunque en aras de la eficiencia los gobiernos han desatado resistencia generalizada y se han desviado de su misión real. Los enfoques convencionales -administración por objetivos, administración por políticas y control total de calidad- tienen grandes restricciones. Frente a ellos se plantean elementos para un enfoque alternativo de la gerencia pública, como gerencia de las interrelaciones organizacionales, y por lo tanto, de la negociación y de la gestión de conflictos; además, la gerencia pública debe centrarse en el verdadero carácter de la eficiencia estatal, asumiendo con Crozier que las organizaciones públicas venden calidad de vida de la población y por lo tanto es necesario invertir a futuro.

Palabras claves: Gerencia Pública, Reforma del Estado, Eficiencia Pública, Racionalidad Política, Política.

Recibido: 15-11-96 - Aceptado: 06-12-96

- Candidato a Dr. en Ciencias Económicas, Director del Departamento de Investigaciones del Centro Interdisciplinario de Estudios Regionales -CIDER- de la Universidad de Los Andes, Colombia. Profesor de Administración Pública y Dirección Universitaria en varios Programas de Postgrado. Ha actuado como consultor en diversos programas de organismos internacionales, entre ellos BM y FAO. Autor de 4 libros y numerosos artículos científicos en revistas internacionales, sobre Estado, Políticas Públicas, Gerencia Pública, Reforma del Estado y Política Energética. 


\section{Public Management under Structural Change Perspectives}

\section{Abstract}

Transformations in business management seem to determine the content and direction of changes in the public sector, and suggest the need for private sector criteria in public management. Governmental changes have centered around decentralization -originating a new territorial concept-, administrative efficiency and for public participation. However, it is not convenient to mechanically transfer models from private business management to the public sector because of their intrinsic differences, due to the dichotomy of politics and management, and the effects this transfer has on the bureaucratic power structure. In public management, political rationality prevails over technical criteria, even when the scenario for change in public administration seems to be free from political content, although in the name of efficiency, governments have unleashed generalized resistance and have deviated from their real mission. The conventional criteria -management by objectives, policy management, and total quality control- have great limitation. An alternative focus for public management is presented here -management through organizational interrelationships, and consequently, management through negotiation and management of conflict; moreover, public management should concentrate on the real character of governmental efficiency, which under Crozier's assumption is that public organizations sell quality of life to their public, and therefore it is necessary to invest in the future.

Key words: Public management, State reform, Public efficiency, Political rationality, Politics.

\section{La nueva vigencia de la gerencia en el sector público}

La dinámica e intensidad de las reformas impuestas por el cambio estructural, requieren del Estado una mayor capacidad de acción y organización. Kliksberg (1984), plantea que esa capacidad debe ser definida como una potencialidad para obtener resultados mediante nuevos tipos de organizaciones en donde es necesario incorporar la óptica "gerencial-empresarial" como principio de manejo.

La progresiva consolidación de la tendencia del Estado a incorporar y reproducir los cambios que estructuralmente han ocurrido en el sector privado, señala la ruta hacia una novedosa vigencia de la Gerencia en el sector público. Desde las tendencias hacia la fusión y adquisición de empresas como alternativa para enfrentar la competencia, hasta las tendencias hacia la descentralización de los procesos productivos, las transformaciones operadas en la empresa parecen haberse convertido en los referentes que marcan el sentido y contenido de las transformaciones del Estado.

En el marco de un traumático ajuste 
en los regímenes fiscal, monetario, cambiario, bancario, laboral, de comercio exterior e inversión extranjera, la tarea de la modernización se reorienta hacia la reforma de la función administrativa del Estado. Precedida unas veces por una reforma constitucional (Colombia, Brasil, $\mathrm{Ni}$ caragua) y otras como antesala a las reformas (Argentina, Venezuela, Uruguay), la reorientación se opera como la creación de las condiciones institucionales necesarias para conciliar funcional, organizacional y administrativamente la eficiencia de las unidades administrativas con la operatividad de las instancias $y$ mecanismos de participación, control y fiscalización ciudadano sobre las burocracias y agencias del Estado.

Los procesos de reforma a la función pública pueden ser resumidos de la siguiente manera: La corporeidad de la acción estatal está definida por una nueva misión, razón y práctica "empresarial" de las relaciones entre la Sociedad y el Estado. Simplicidad de las estructuras y procedimientos y autonomía de las unidades operativas, se invocan como los ejes de una acción pública selectiva, flexible y eficiente. (Medellín Torres, 1992a)

Sin duda, América Latina está transitando por un proceso de transformación que procura el paso de una gestión estática de recursos hacia una gestión dinámica del saber y la destreza social. La gestión pública está dejando de ser "encapsulada" en la concepción fija de la intervención extensiva y centralizada del Estado, para convertirse en la expresión del saber-hacer de los individuos y las organizaciones sociales. (Medellin Torres, 1992b)
De una percepción rígida de entidades y organismos territoriales y sectoriales, entramos en la concepción flexible de las empresas públicas, que se estructura desde una relación jerárquico-funcional del trabajo y el poder, para entrar a alimentarse de un trabajo profesionalizado (despolitizado) que, como fundador del valor económico, es constantemente renovador y por eso ya no debe ser más asimilado a un costo sino como un soporte de la inversión empresarial del Estado. La difusión de principios tales como costo-beneficio, calidad de la prestación del servicio, información al cliente y el concepto global de servicio, reconvierten las estructuras y organizaciones en empresas públicas, a sus gobernantes en gerentes y a sus usuarios en clientes. (Medellín Torres, 1992c)

La vieja oposición entre saber muerto y saber vivo, propio de las estructuraciones de los años sesenta y setenta, cede su lugar a una nueva visión, al desarrollo de saberes vivos y al manejo estratégico de los recursos. Desde este punto de vista, una intervención extensiva y repetitiva, no calificada ni especializada, ejecutada en aplicación de normas y regulaciones estáticas, se constituye en un saber muerto. La fuente esencial de recuperación para la "empresa pública" se desplaza de los saberes muertos (extensión y centralización) a saberes vivos (destrezas, capacidades de confrontar la competencia).

Pero el desplazamiento hacia los saberes vivos no puede darse sobre el libre arbitrio de gestión de las empresas públicas. Tal como lo afirma Crozier: 
"Una empresa pública es competitlva sólo a condlción de que promueva la callficaclón de los recursos humanos, se informe sobre su localizaclón estratéglca y sobre las tendenclas de los mercados (político y económico) y desarrolle una acción flexlble y comprehensiva en su esfera de acclón".

Su nuevo carácter como "empresa" pública dependerá de reestablecer el equilibrio entre dos legitimidades. Por una parte, su legitimidad patrimonial que se nutre de las contribuciones, aportes y pagos públicos. Y por otra, la legitimidad de sus competencias (que no es la legitimidad politica), que se nutre de la eficiencia económica sobre el poder operacional que su gestor (gerente) le confiera. (Lorino, 1991)

En este contexto, la administración del desarrolio enfrenta nuevos escenarios, cada vez más complejos y más conflictivos. Por una parte, confronta una novedosa territorialidad de las relaciones y procesos sociales. Las mediaciones tradicionales del Estado-Nación que sustentaban el ordenamiento territorial y financiero, para la asignación y uso de los recursos públicos, han perdido vigencia. En su lugar emergen nuevas mediaciones político-institucionales, burocráticoadministrativas y económico-sociales, en donde la magnitud, intensidad y dinámica de los procesos sociales participativos y las relaciones de mercado, son las que progresivamente definen el sentido y contenido del ordenamiento estatal en la asignación y uso de los recursos públicos.
Por otra parte, la administración del desarrollo confronta una nueva institucionalización del orden social. Los principios organizacionales y las estructuras jerárquicas del poder que sostenian tradicionaimente la acción de las políticas públicas, se han quebrado frente a una nueva dinámica de relación funcional de las instítuciones estatales. La descentralización de funciones y la destreza empresarial de los tomadores de decisiones en la conducción de los procesos sociales, abren un nuevo escenario a los principios organizacionales de la función pública, a la estructura empresarial de dirección y gestión de las políticas públicas, y a las estructuras jerárquicas del poder, esto es, estructuras decisionales compartamentalizadas por objetivos y relaciones conflictivas.

\section{Disyuntivas y condicionantes de la gerencia en el sector público}

Los cambios en la función pública parecen sugerir que los enfoques, modalidades y formas de administración y gestión de las organizaciones privadas pueden ser aplicados indistintamente en las entidades y organizaciones públicas. En todos los ámbitos de la acción pública, los procesos administrativos, la orientación del desarrollo, el financiamiento de las políticas públicas, el acceso a la información, el control y fiscalización ciudadana - aún la distribución del ingreso, aparecen cada vez más subordinadas a la aplicación técnica de conceptos y prácticas de calidad total, gerencia del servicio, planificación estratégica, administración 
por objetivos, etc. Asi mismo los cambios en las estructuras, funciones y procedimientos que se han venido aplicando a las entidades y organizaciones públicas, han comenzado a estar guiadas por criterios y principios organizacionales propios del sector privado.

Sin embargo, la existencia misma de factores tales como la mayor complejidad organizativa y operacional, la interferencia de intereses politicos, la fragilidad de los procesos decisionales o la diferencia cualitativa entre lo que significa producir bienes y servicios públicos y producir bienes y servicios privados, no sólo cuestiona la consistencia misma de tales conceptos y prácticas en aplicación directa y mecánica al sector público, sino que más estructuralmente plantea la inconveniencia de "transferir" mecánicamente enfoques, modalidades y formas de administración y gestión de las organizaciones del sector privado al sector público.

A pesar de la novedosa naturaleza "empresarial" que el cambio estructural le confiere a las entidades y organizaciones públicas, (Case, R. H. 1996), la aplicación de los elementos de administración y gestión privada al sector público confrontan tres tipos de restricciones, relacionadas entre sí:

a. La diferenciación misma de la naturaleza y funcionalidad entre sector público y sector privado;

b. La diferencia que, sobre las estructuras, funciones y procedimientos de las organizaciones públicas, impone la existencia de la dicotomía políticaadministración;

c. El peso específico que sobre la gestión y administración ejercen las burocracias en el comportamiento orga- nizacional y en la obtención de resultados.

\section{- Sector público y sector privado}

En términos de la naturaleza y funcionalidad, entre el sector público y el sector privado, existen tres diferencias cruciales.

La primera proviene de la ausencia de la noción de mercado y competencia en el sector público (Crozier). En la mayoría de los casos, los éxitos o fracasos de las entidades y organizaciones públicas no sólo están desconectados de las presiones o cambios que los competidores le pueden imponer en la producción y realización "mercantil" de sus bienes o servicios. Más profundamente el diseño y operación de las estructuras, funciones y procedimientos organizacionales, responden a una lógica jurídico-institucional y no a los requerimientos impuestos por una particular dinámica de mercado.

En primer lugar, la permeabilidad del interés colectivo por los intereses particulares (por ejemplo, de la acción burocrática en la asignación y uso de los recursos), imprime una complejidad tal que los objetivos organizacionales se multiplican y entrecruzan en un cuadro que debe articular no sólo dinámicas sectoriales con dinámicas territoriales, sino conciliar necesidades con disponibilidades en un marco altamente expuesto a una multiplicidad de presiones.

En segundo lugar, la ausencia de un principio de exclusión de los bienes públicos, que resulta del curso "forzoso" en la provisión de bienes y servicios bajo estructuras monopólicas y del carácter 
estrictamente juridico-funcional que rige el control y la fiscalización sobre la acción pública, no le permite a las empresas públicas conocer las preferencias de los "consumidores", ni identificar problemas de calidad en la producción de sus bienes y servicios de un modo tal que les posibilite corregir los problemas y responder más rápidamente a las exigencias de los "mercados".

En tercer lugar, la existencia de rígidas estructuras de administración pública y el apego a los formalismos y regulaciones, no sólo obstruyen la acción rápida, flexible y eficiente de las organizaciones públicas, sino que también distorsionan y obstaculizan el establecimiento de sistemas y parámetros de eficiencia, calidad y rendimiento que permitan evaluar comparativamente la producción de bienes y servicios públicos con los bienes y servicios que se venden en los mercados convencionales.

La segunda diferencia entre sector público y privado, radica en el hecho de que en las organizaciones públicas, son la naturaleza intrínseca de la organización y la base tecnológica de su actividad las que tienden a definir de antemano su comportamiento. Recientes estudios han demostrado como, al requerir una determinada disciplina organizativa y operacional, las actividades técnicas en sectores como la industria, el transporte aéreo, las telecomunicaciones $y$, en algunos casos la electricidad, han podido mantener, incluso en condiciones económicas muy adversas, un cierto nivel mínimo de eficiencia operativa. Esto se debe no sólo a que su naturaleza funcional le permite definir sus frentes de acción especifica y con ellos sus objetivos, estrategias y metas de corto plazo, sino que también las tecnologías avanzadas que debe utilizar requieren de una mayor disciplina operativa y organizacional". (Israel)

Por el contrario, en las organizaciones públicas que se desempeñan principalmente en los llamados sectores 'sociales', las características intrínsecas de sus acciones no les permiten definir especiffcamente ámbitos permanentes de trabajo, con lo cual les resulta bien difícil definir objetivos precisos y metas de corto plazo. Además, la base tecnológica de sus actividades requiere de poca disciplina y no son lo suficientemente especificas. En los hechos, la diversidad de objetivos y funciones en materia de política social hace que, a diferencia de las actividades técnicas, la disciplina organizacional y operativa de las actividades sociales esté permanentemente sometida a los cambios de prioridades que producen los cambios en las agendas de gobierno o la necesidad de responder a una situación contingente.

La diferencia, en términos del comportamiento organizacional, se traduce en estructuras de costos crecientes y bajos niveles de productividad, que no sóio generan inequidades en la distribución de los recursos, sino que además, como lo plantea Cárdenas (1990), desatan una aguda competencia por recursos públicos en el largo plazo ante la creciente dificultad de obtener recursos suficientes para la financiación sectorial.

La tercera diferencia está constituida por los muy distintos niveles que, en términos de los márgenes de maniobra, existen entre los empresarios públicos y 
los empresarios privados en la dirección y posicionamiento de sus organizaciones.

Majone (1992) demuestra cómo la creencia generalizada de que "Los hombres de gobierno logran sus propios objetivos mediante la ejecución de las acciones gubernamentales que más complacen al electorado, del mismo modo que los empresarios privados obtienen ganancias produciendo lo que la gente desea, parte de un supuesto falso". Se considera equivocadamente que, asi como el empresario privado tiene completa libertad para modificar sus niveles de producción y costos en aras de mayor ganancia, el empresario público tiene un manejo discrecional para definir los objetivos de la organización o para asignar los recursos en el sentido que él desee (Israel, 26).

En su argumentación, Majone muestra cómo en las organizaciones públicas la posibilidad de sustituir un insumo por otro, con el propósito de adecuarse a determinadas circunstancias y requerimientos del consumo colectivo, está severamente limitada por la interferencia de intereses políticos, prácticas burocráticas y disponibilidades fiscales. Esta situación implica que, por una parte, el proceso presupuestal adquiere un carácter esencialmente político, en la medida que la acción pública está subordinada al logro de determinados objetivos en aquellos frentes que reporten "rentabilidad" buro- crática y electoral. $Y$, por otra, que en el proceso de definición y asignación de las políticas y los recursos públicos, el criterio de "factibilidad" tenga muchos más elementos de validez y realidad que el criterio de "optimalidad" de las políticas. (Majone, 1992)

Así mismo, existen otros elementos que contribuyen a mantener bajos márgenes de maniobra organizativa y operacional de los empresarios públicos. Entre ellos se pueden considerar las presiones que sobre la toma de decisiones imponen las estructuras y sistemas de administración y remuneración del personal. Por una parte, el carácter político en el manejo de las burocracias se traduce en una precaria estabilidad de empleados y trabajadores en los niveles directivos y en los operativos. Mientras que en el sector privado las directivas tienden a permanecer y sus equipos de operarios a cambiar según las necesidades productivas y los requerimientos del mercado, en el sector público son los trabajadores del nivel operativo los que permanecen y sus directivas las que presentan un elevado nivel de rotación. Por otra, las presiones que sobre los niveles de remuneración al trabajo ejercen los desajustes presupuestales y financieros, se revierten en la imposibilidad de establecer estructuras y sistemas de remuneración que estimulen la productividad del trabajo. En los hechos, las diferencias salariales entre el sector público y el sector privado distorsionan la

El equivoco se fundamenta en afirmaciones tales como aquella que desarrolla Arturo israel en el sentido de que "las empresas públicas fracasan porque los hombres de gobiemo son incapaces de ofrecer resistencia a las interferencias políticas, a pesar de todas las protecciones que se incorporen al sistema y no porque ellas estén desprovistas teóricamente de la capacidad para lograr una operación eficiente, pág. 126. 
movilidad de los factores y con ello la asignación de recursos en el mercado hacia el sector privado.

\section{- Política y administración}

Sin duda, la transferencia directa de enfoques, modalidades y formas de administración y gestión de las organizaciones del sector privado al sector público, encuentra en la dicotomía política-administración uno de sus principales escollos. Desde los trabajos pioneros de Goodnow y Wilson, que difunden un sistema analítico en donde es una dirección politica electa popularmente la que dicta las ordenes y un aparato administrativo el que las ejecuta ${ }^{2}$, hasta los recientes aportes de Crozier y Kliksberg, que desarrollan análisis que integran dirección política y aparato administrativo, la dicotomia entre política y administración aparece como un elemento distintivo, propio y particular de los procesos y estructuras organizacionales del sector público.

En el marco de una progresiva irrupción del intervencionismo estatal, la expansión burocrática y la novedosa imbricación entre sector público y sector privado, se abren intensos debates en torno a la validez y consistencia de la dicotomía Política-Administración como factor explicativo del comportamiento del sector público. La evidencia de una creciente interferencia de las relaciones de poder al interior y exterior de las organizaciones y procesos administrativos, deja entrever la existencia de límites al desempeño de las organizaciones públicas.

Por una parte, bajo el referente de la "interrelación entre roles políticos y roles administrativos", los defensores de la separación Política/Administración argumentan la pertinencia y consistencia de la dicotomía. Oscar Oszlak (1989), para citar un ejemplo, desarrolla dos modelos de acción-decisión que distinguen la racionalidad técnica (modelo de intelección) de la racionalidad política (modelo de interacción), para demostrar cómo la for * mulación e implementación de políticas públicas son, alternativamente, ámbitos separados y yuxtapuestos de reflexión y acción. Sostiene Oszlak que los límites entre política y administración están determinados por la distinta naturaleza de las interdependencias existentes entre los agentes y unidades estatales que intervienen en los procesos de decisión y ejecución de las políticas.

Por otra parte, los enemigos de la separación entre política y administración, se ubican en el extremo analítico que desliga los procesos de formulación y los procesos de implementación de poIíticas, para plantear que tal separación no es otra cosa que el producto de:

a. Un espejismo institucional, que al imputar las responsabilidades de la política a una determinada institución, desconoce las implicaciones de la relación que existe entre la acción decisional y la acción administrativa;

b. La incapacidad analitica para captar propio de la política. Las cuestiones administrativas no son cuestiones politicas. Aunque la política fija las tareas de la administración debe abstenerse de manipular sus oficinas. (Cit. por Oszlak, 1989). 
fenómenos dinámicos que convierten las políticas en acciones;

c. La distinción analítica necesaria para la comprensión de los fenómenos públicos.

Quizá uno de los más contundentes argumentos de los enemigos de la separación entre Política y Administración, consiste en que la no consideración de los problemas políticos en los análisis de las organizaciones conduce a visiones parciales. El estudio pionero de Crozier (1963) sobre las organizaciones públicas francesas, sirvió de base para demostrar como los distintos tipos de lucha por el poder que tienen lugar interna o externamente a una organización, pueden llegar a constituirse en uno de los más importantes factores explicativos del bajo rendimiento de las organizaciones del sector público ${ }^{3}$.

No son pocos los estudios que, posteriormente, han demostrado cómo las interferencias politicas hacen que los aparatos burocráticos lleguen a tener tal fuerza que, en muchas ocasiones, sea el propio aparato el que determina las decisiones de los altos niveles. Para Kliksberg, por ejemplo, las interferencias tienen un peso tan grande en el comportamiento de las organizaciones, que las alternativas que llegan a las cumbres del poder, en términos del árbol de decisio- nes organizacionales, suelen estar configuradas desde abajo y no desde arriba. Esto explica su conclusión de que el fenómeno es tan complicado que exige que los "políticos" manejen políticamente la relación con el aparato público.

No obstante, estos análisis parecen haber perdido de vista la esencia y componentes diferenciales que desde la política interfieren en el comportamiento de las organizaciones. En los hechos, las tensiones y conflictos de poder, al interior o exterior de las organizaciones, si bien pueden llegar a proyectarse como un problema de naturaleza política, esto no significa que necesariamente se constituyan en un problema político o de "la política". Esto es, que en su explosión, manifestación y soluciones, las confrontaciones entre distintas fuerzas pueden presentar rasgos distintivos de una situación política (que puede resolverse o no "politicamente"), pero sin llegar a tener el carácter de un problema político ${ }^{4}$. De hecho, si se quiere, todos y cada uno de los problemas que confrontan las organizaciones pueden tener una interferencia "última" de la política. Pero no por ello se supone que un buen "manejo" de los políticos resuelva los problemas.

La separación entre política y administración, radica en los ámbitos mismos en los que cada una se desenvuelve. Por

3 En su investigación, Crozier encuentra cómo entre los conflictos hay dos corrientes de presión y ambas se orientan a normar la organización. Los niveles superiores quieren normar las tareas, mientras que los de abajo quieren normar las remuneraciones, los sistemas de ascenso, etc. Citado por Klisberg (1990).

4 Asi por ejemplo, se puede tratar de un problema que por sus dimensiones es de naturaleza económica (cuando se trata de definir salarios, ascensos, asignación presupuestal, provisión de los recursos requeridos, etc.); jurídico-administrativa (cuando se trata de problemas de superposición o colisión de funciones y competencias); social (cuando se trata de problemas relacionados con el abastecimiento de bienes básicos), etc. 
una parte, la Administración es el proceso mediante el cual se busca crear y mantener el ambiente organizacional, funcional y procedimental necesario para el desempeño eficaz y eficiente de los individuos en la aplicación de una tarea puntual (generalmente repetitiva y producto de un acuerdo mutual de relación salarial), por arreglo a una función específica y en cumplimiento de una misión organizacional definida. Varios elementos caracterizan la administración y al administrador. En primer lugar se trata de un problema de asignaciones. No de otra manera encuentra sentido la existencia de sus instrumentos tradicionales como planeación, organización, nombramiento de personal, dirección, control, etc. Su naturaleza está definida por verdades fundamentales de validez general para cualquier caso y escala que se trate. Sus metas y objetivos son verificables, tangibles $y$ mensurables. En segundo lugar, su esencia es la estabilidad. Su propósito está definido de antemano y no puede ser el producto de un conjunto de acciones contingentes, descoordinadas, ni desatadas por presiones externas de cualquier carácter. $Y$ en tercer lugar su móvil es la generación de excedentes.

Por otra parte, la Política es el conjunto de estructuras y prácticas a través de las cuales no sólo se imprime una determinada dirección ideológica, económica y social a una sociedad, por encima de una diversa gama de alternativas. También es el ámbito en donde se desata una particular dinámica relacional que cohesiona a los individuos en torno a un conjunto de principios y tradiciones, moviliza recursos, activa movimientos por arreglo a un conjunto de intereses y necesidades conflictivas y contradictorias entre si. A diferencia de la administración, su naturaleza no está definida por verdades fundamentales para cualquier caso y escala que se trata. Está definida por un conjunto de principios y valoraciones cuya relatividad depende del contenido ideológico conque los individuos se posicionen ante una situación determinada. Sus metas y objetivos son parcialmente verificables, la mayoría no tangibles ni mensurables. Su propósito no está definido de antemano, puede depender de un conjunto de acciones contingentes y descoordinadas, desatadas por presiones externas de cualquier tipo. Su campo de acción tiene lugar en términos de la relación Estado-Sociedad, su móvil es el cambio político y su característica, la de trascender hacia la definición del orden social.

Recuperando la versión clásica sugerida en 1900 por Frank Goodnow y Woodrow Wilson, en el sentido de que "Los funcionarios electos (aquellos a los que el voto confirió poder deberían hacer la política, mientras que la burocracia deberia mantenerse por fuera de la toma de decisiones y atenerse a administrar en forma competente las decisiones políticas", la separación entre política y administración, más que una separación de responsabilidades, como la identifica Kelman, expresa una diferenciación de escenarios de acción en donde (Medellín Torres, 1993):

a. El conflicto, como esencia de la política, expresa los grados de confrontación entre actores con tramas, intereses y racionalidades bien distintas (en unos casos irreconciliables) en 
función de un objetivo particular e independiente de los demás;

b. La incertidumbre, como terreno natural de la política, señala los grados de tensión y la intensidad con que los conflictos llegan a permear las acciones, afectos y procedimientos de los individuos en su vida personal y colectiva;

c. El poder que, como móvil de la politica, expresa la capacidad para afectar el sentido y contenido de las decisiones políticas y de política, así como para definir el rumbo de las acciones estatales y gubernamentales.

En términos del análisis de los procesos organizacionales en el sector público, la separación entre política y administración tiene significativa vigencia en la medida que permite:

a. Delimitar los campos que separan la función pública de la función estatal. Mientras que los trazados de política pública implican estructuras, funciones y procedimientos que, aún pudiendo ser prestados por agentes privados, mantienen su carácter público, los trazados de política estatal implican estructuras, funciones y procedimientos que van más allá de los gobiernos y sólo pueden ser prestados por el propio Estado;

b. Delimitar los alcances e identificar los criterios con que avanzan los procesos de institucionalización de las polf́ticas y organizaciones públicas. Es decir, de los procesos mediante los cuales unas determinadas organizaciones adquieren un valor y estabilidad específica tales que les confiere grados de estatidad por sobre otras organizaciones ${ }^{5}$.

\section{- Burocracia y poder burocrático}

Sin duda, uno de los factores explicativos de los desatamientos de conflictos de poder que interfieren los procesos administrativos y afectan los rendimientos de la organización pública, lo constituye el llamado "poder burocrático". Lejos de las visiones instrumentalistas que han caracterizado los análisis del fenómeno burocrático en América Latina ${ }^{6}$, (Márquez y Godan, 1992) el concepto de poder burocrático está asociado al de un "leviatán" (fuerza política autónoma) que, teniendo como función primordial la de traducir las políticas y estrategias giobales en acciones por planes y proyectos

Samuel Huntington, (1991) afirma que "una organización capaz de adaptarse a los cambios producidos en su ambiente y que ha logrado sobrevivir a uno o más de ellos en sus principales funciones, está más institucionalizada que otra que no pasó por esa experiencia. La adaptabilidad da la medida cabal de una organización altamente desarrollada. La institucionalización la convierte en algo más que en un instrumento para ciertos fínes..."

La concepción instrumental de la burocracia en América Latína ha transitado desde las visiones weberianas, que despojan al poder de su contenido coercitivo y lo definen como el derecho al ejercicio de una autoridad técnicamente fundamentada y limitada, pasando por las lecturas personianas que, con esquemas sistémicos, definen a la burocracia como la capacidad de movilizar recursos para desempeñar las funciones propias del sistema organizacional, hasta los enfoques que siguen a Crozier, que se interesan por las disfunciones de la eficiencia de los modelos organizacionales y las definen como patologías que se desplazan entre el formalismo, la excesiva jerarquización y la corrupción. 
puntuales, tiende a constituirse estructuralmente en un factor de interferencia y bloqueo a la acción de las organizaciones en el cumplimiento de sus propósitos.

En el marco de un progresivo reforzamiento de la regulación y control fiscal y administrativo, la acción de las organizaciones públicas se caracteriza por la presencia permanente de luchas de poder. Es la lucha por la mayor autonomia y un menor control, en donde la organización busca imponer sus prerrogativas de una manera tal que le permita no sólo subordinar a las demás organizaciones en torno suyo, sino también lograr que sus acciones sean las que le impriman un rumbo definido a los procesos institucionales de dirección política, económica y social, en una sociedad. De esta manera, las organizaciones desarrollan estrategias que aseguren, por una parte, la certidumbre en la expedición de disposiciones protectoras y mando sobre el personal y, por otra, la extensión de los ámbitos de competencia que les permitan absorber recursos y generar una privilegiada posición institucional en el trazado de las políticas públicas.

En la perspectiva de las transformaciones del Estado y la función pública, la dinámica del cambio estructural se proyecta tradicionalmente como un revuelta contra las formas jerárquicas y funcionales de poder heredadas del pasado. Expuestas a las presiones del cambio, cada nicho de poder organizacional y burocrático explota en defensa de sus propias prerrogativas. Invocando la defensa de lo establecido o por la modernización, irrumpen en escena cobrando vida propia con el propósito de modificar en su favor la correlación de fuerzas existentes. Arrojadas al vacío de los desequilibrios y el desorden, las estructuras estatales quedan expuestas a la intensidad de los combates internos que desata cada iniciativa. Una multiplicidad de inesperados actores pugna buscando delimitar y controlar el poder en su propia parcela. (Medellín Torres, 1992b)

La dinámica de la confrontación hace que las luchas y tensiones se desplacen de las organizaciones hacia las burocracias que las componen y viceversa. El poder burocrático se define, siguiendo a Brown y Erie, como una relación asimétrica de dependencia entre dos o más actores intra e inter organizacionales, que en presencia de conflictos adquiere caracteres unidimensionales cuando se trata de una problemática puntual, o caracteres estructurales cuando el conflicto está inmerso en el sistema y sus instituciones políticas, económicas y sociales. (Brown y Erie, 1984). La lucha por el poder burocrático se desarrolla como una lucha desatada por el control político y funcional, en donde la formalidad institucional, los ámbitos de competencia y los vinculos externos juegan un papel clave en la definición del conflicto.

El poder burocrático, más que un poder de feudo, es el poder de la información y el manejo de los recursos. Tanto el que fluye desde el exterior hacia el interior de las organizaciones (en el despliegue de las políticas y estrategias), como el que fluye desde el interior hacia el exterior de las organizaciones (en la aplicación de recursos, competencias, proyectos). En un escenario característico de lucha por el poder burocrático, la toma de decisio- 
nes aparece determinada por el secreto y la connotación militar de las intervenciones. Por el secreto, porque es el medio a traves del cual se busca imponer la primacía del carácter individual de intereses burocráticos y organizacionales particulares, sobre el carácter colectivo de los intereses burocráticos y organizacionales generales. Por la connotación militar, porque es el medio táctico (en el sentido militar del término) a través del cual quienes toman la decisión en secreto tratan de identificar y evaluar los frentes posibles de tensión y conflicto social o institucional que desata o puede desatar una decisión. En un escenario decisional minado por éstos dos factores, las llamadas de prestigio desplazan a las llamadas de función o jerarquía. La movilización de fuerzas efectivas supera las investiduras institucionales como muestra de poder.

El poder burocrático se proyecta como recurso de bloqueo. Como fuerza de resistencia al cambio que se aplica, en unos casos, como factor de desplazamiento de los objetivos (de los formales a los coyunturales), que explica su poder como una respuesta a los inciertos y hostiles escenarios políticos y su acción como una estrategia para asegurar la supervivencia (Brown y Erie, 1984). En otros, como factor de conflicto, que explica su poder como el resultado del intento de los intereses dominantes por continuar con el control del Estado y las políticas públicas, y su acción como el desplazamiento de la formulación de planes y políticas hacia agencias administrativas dependientes para neutralizar los desafíos y presiones de los intereses "enemigos".

El poder burocrático, lejos de cons- tituir una amenaza para la "estabilidad" del orden institucional establecido, se constituye en una estrategia consciente de política que utilizan burócratas y politicos para compensar los "desajustes" que la lucha de poderes desata y para contener los desarreglos y rupturas que la dinámica del cambio genera en el desenvolvimiento de las fuerzas inerciales en las organizaciones.

\section{La especificidad de la gerencia en el sector público}

Puesta en el marco del cambio estructural, la incorporación de la práctica "gerencial-empresarial" como principio de manejo de la función pública, comporta, siguiendo a Oscar Oszlak, un supuesto fundamental. "La gerencia presupone que la acción administrativa puede $\mathbf{s u -}$ bordinarse a la razón, y que el comportamiento de las estructuras y organizaciones públicas puede dirigirse hacia el logro de determinados fines, eligiendo para ello los medios más racionales". De hecho esta lectura supone que el Estado es neutro y que el aparato estatal y las organizaciones públicas que lo conforman están desprovistos de intereses, que hay perfecta sincronía entre medios y fines, $y$ que la información es un recurso abundante y disponible.

Sin embargo, la dinámica transicional hacia un Estado más democrático y eficiente debe conducir a que la llamada gerencia para existir, debe presuponer que la acción administrativa es más bien el producto de transacciones entre partes (arreglo y conciliación de intereses) y que la consecución del interés individual 
de cada una busca realizar el fin colectivo que las vincula. Esta lectura alternativa presupone que el Estado no es neutro; que el aparato estatal y las organizaciones que lo componen encarnan la permeabilidad del interés público por el interés privado; que no existe una relación de sincronía entre fines y medios y que la información es un recurso escaso y fuente de poder. La operación sobre el conflicto tiende a develar intereses en juego, agendas ocultas, descubrir y desarticular alianzas no deseadas.

Mientras que en el primer caso la racionalidad técnica tiende a primar sobre la racionalidad política, en el segundo pareciera primar una visión hacia la tendencia contraria. Esto es, que en el escenario transicional la racionalidad politica tiende a primar sobre la racionalidad técnica. Sin embargo, esta consideración es cierta pero solamente de manera aparente. En un escenario de cambio, la acción gerencial pública pareciera suponer una acción vacía de contenido político y llena de contenido técnico, aunque también se podría afirmar que en el mismo escenario la gerencia no es otra cosa que una acción disfrazada de la política (por un recurso técnico) para restablecer la legitimidad de la acción estatal en la sociedad o para recuperar los lugares perdidos por el Estado, a causa de su "ineficiencia".

Faulkner afirma en un reciente ensayo, (1992), cómo, en la perspectiva del cambio estructual, los gobiernos han sido, en aras de la eficiencia, muy acuciosos despidiendo empleados, reduciendo el tamaño del Estado e incursionando en la aplicación y revisión de programas de privatización. Pero los procesos de adap- tación institucional al cambio no sólo están desatando resistencia generalizada, sino, lo que es peor, está conduciendo las reformas por un camino equivocado.

Sostiene Faulkner, que en la medida en que la gerencia del sector público se concentra en mejorar la eficiencia dentro de las organizaciones gubernamentales, se está desviando de su misión real. La propuesta de Faulkner apunta a que los conceptos, enfoques y modalidades de gerencia que se proponen para el sector público no se deben encontrar en la empresa privada, debido a que la importancia y capacidad del sector privado es siempre limitada y que lo que encara el sector público es una exigencia innovadora fundamental que modifique los esquemas demasiado dirigidos, excesivamente gobernados por reglas, demasiado jerárquicos e inclinados en exceso a interiorizar mecanismos de control.

En el ámbito de las relaciones intergubernamentales, terreno on el que Faulkner hace su llamado, es claro el hecho de que la racionalidad técnica y la racionalidad política se articulan, siempre de manera dinámica y conflictiva, en función de una determinada dirección e intensidad de los procesos institucionales que tienen lugar en las organizaciones públicas, en torno a los fenómenos políticos, económicos y sociales de una sociedad. Se trata de procesos siempre interdependientes en donde las acciones de cada una de las partes (cada organización del sistema con sus propios actores, racionalidades y conflictos), afectan los comportamientos y resultados del conjunto del aparato estatal y las organizaciones públicas. 
En este escenario aparece claro un necho:
"el desempeño cualitativo y cuantitativo de las organizaciones públicas, a dife- rencla de las organizaciones privadas, tiene lugar en macrosistemas y bajo principios y restricciones de interrela- ciones organizacionales"

La gerencia para el sector público, debe hacer referencia al conjunto de conceptos, enfoques y modalidades de gerencia que potencien y desarrollen la habilidad para manejar las interacciones entre entidades públicas, y las relaciones entre niveles de gobierno y entre éstos y el sector privado. Sin duda, la especificidad de la Gerencia Pública consiste en realidaden la Gerencia de las interdependencias Organizacionales. (Faulkner, 1992)

Plantear que la gerencia pública consiste en la gerencia de las interdependencias organizacionales, tiene dos significativas implicaciones. La primera gran implicación es que la gerencia pública adquiere sentido $y$ contenido es en el proceso de trazado de políticas públicas. Es el trazado de las políticas públicas, el que define el lugar y la ruta critica de la gerencia en el sector público. La segunda implicación consiste en que el establecimiento del equilibrio organizacional, entre legitimidad patrimonial y legitimidad de competencias, tiene en la negociación y gestión de conflictos al fundamento más primario e importante de la gerencia pública. Detengámonos por un momento en éstas implicaciones.
- El trazado de las políticas públicas: hacia una definición del lugar de la gerencia en el sector público

En torno a la primera implicación, vista en el contexto de la acción organizacional pública, la racionalidad técnica y la racionalidad política se encuentran en el trazado de políticas públicas, que es el proceso en donde también se confrontan y cumplen administración y política. Es decir, el proceso mediante el cual las estructuras, funciones y procedimientos propios de la administración interactuan con un determinado conjunto de estructuras y prácticas políticas de dirección ideológica, institucional, económica y social. En este sentido, el trazado de políticas públicas puede ser definido:

a. Como un proceso de sentido. Es decir, un conjunto dinámico de acciones propositivas, intencionales, planeadas y no reactivas, que se pone en movimiento con la decisión de alcanzar ciertos objetivos a través de ciertos medios;

b. Como un curso de acción que involucra todo un conjunto complejo de decisores y operadores, más que a una acción suprema e instantánea. Es, por tanto, el curso de acción deliberadamente diseñado y el curso de acción efectivamente seguido;

c. Como una acción contingente a la que se recurre de manera parcial y desarticulada como un mecanismo puramente reactivo a las situaciones y problemas que se desatan y explotan poniendo en cuestión el orden establecido. 
Puesta en términos de los procesos organizacionales del sector público, la toma de decisiones, su implementación y evaluación, se desplazan entre tres tipos distintos de trazado de política pública:

a. Trazado Político, caracterizado por ser un modelo prescriptivo de un proceso de sentido que busca seguir un gobierno durante un período de tiempo definido. Mediante un proceso que comporta la ruta crítica: Idea Política-Elección/ Política-Producción/ Políticas-Medición de Resultados, se busca definir unos objetivos precisos con metas específicas. Parte del supuesto de que el gobernante tiene el poder institucional, el control funcional y la información disponible para liderar el comportamiento organizacional. De alli se deduce que los objetivos de las organizaciones se pueden subordinar a los objetivos del gobernante;

b. Trazado Técnico, caracterizado por ser un modelo descriptivo del curso de acción que siguen los tomadores y operadores de las decisiones de política pública, en busca de unos resultados de solución a problemas estructurales y a situaciones de coyuntura. Mediante un proceso que comporta la ruta crítica: GestaciónFormulación - Implementación - Evaluación de Políticas, el modelo técnico busca identificar y procesar los ejes problemáticos que debe enfrentar la acción institucional del Estado. Al igual que el modelo político, el modelo técnico presupone que existe total control, permeabilidad y liderazgo en el aparato estatal, con la diferencia de que están referidos a la primacía de la racionalidad técnica sobre los intereses políticos;

c. Trazado Político-Espasmódico, caracterizado por ser un modelo impositivo de carácter coyuntural, una acción suprema e instantánea de gobierno en el enfrentamiento de aquellos problemas y situaciones contingentes que al aparecer tienden a desestabilizarle y a fracturar la organización y operación del aparato público. Mediante un proceso que comporta la ruta secuencial y sucesiva de accióndecisión, en donde una acción conduce a una decisión que, a su vez, conduce a una nueva acción y ésta a una nueva decisión, el modelo político-espasmódico busca generar impactos inmediatos, resultados concretos. Partiendo del supuesto de que el poder central no tiene el control sobre el aparato público, y que las organizaciones responden a intereses fragmentados y particulares, las políticas se formulan e implementan sólo por referencia a una situación presente. No hay perspectiva histórica y visión del futuro.

En las organizaciones públicas, la dinámica decisional que rige los trazados de política pública está determinada por la configuración de las estructuras jerárquicas del poder, las relaciones de dependencia funcional entre las organizaciones $y$, en alguna medida, las exigencias del momento. Los trazados de polítca pública no solamente expresan los grados relativos de ascendencia y control político, que tienen los gobernantes sobre 
el aparato público, sino que además reflejan los niveles de tensión y conflicto que existe entre las entidades y organizaciones que lo componen.

El Estado en tanto macro-organización, se proyecta como una gran entidad corporativa, en cuyo interior la investidura jerárquica, la naturaleza institucional, el rol orgánico-funcional y los niveles de conflicto burocrático, interno y externo, definen una clara segmentación sectorial y territorial de los trazados de política pública:

a. Entre el proceso de toma de decisiones y el proceso de definición y formulación global de las políticas y estrategias, a través de las cuales se imprime un sentido y contenido que oriente las decisiones, para cada conjunto de entidades y organizaciones sectoriales y territoriales del aparato público;

b. Entre el proceso de definición y formulación de las políticas y estrategias y el proceso de despliegue de tales políticas y estrategias, ya con sentido y contenido específico para cada organización en su campo particular de desempeño;

c. Entre el proceso de despliegue de las políticas y estrategias de cada organización y el proceso de "desborde", de las acciones y operaciones organizacionales, sobre los usuarios y beneficiarios de sus programas y proyectos puntuales.
Puesta en estos términos, la dinámica decisional de las organizaciones implica que la gerencia pública, como gerencia de la interrelación de las organizaciones, tiene lugar en los elementos que conectan los procesos de formulación de las políticas y estrategias y los procesos de despliegue y de desborde de tales políticas y estrategias. Estos conectores comprenden actividades tales como la planificación, coordinación, ejecución, evaluación y mejoramiento. Este es el campo especifico de la gerencia en el sector público.

\section{- La negociación y gestión de conflictos como fundamento de la gerencia en el sector pú- blico}

La segunda gran implicación de aceptar que el objeto de la Gerencia Pública es la Gerencia de las interdependencias organizacionales, radica en que el establecimiento del equilibrio organizacional entre legitimidad patrimonial y legitimidad de competencias, tiene en la negociación y gestión de conflictos a su fundamento más primario e importante. La negociación es entendida como el proceso mediante el cual dos o más partes en conflicto analizan y determinan las condiciones requeridas para llegar a un acuerdo $^{7}$. (Bartos, 1985) La gestión, por otra parte, es definida como el proceso en

7 Vale la pena aclarar que la negociación no es el acuerdo. El simple hecho de que dos o más actores en conflicto se sienten en una mesa a considerar las posibles condiciones de arreglo a sus diferencias, ya se constituye, al menos en un principio de negociación. 
donde las partes en conflicto identifican y seleccionan una ruta crítica de acciones para el cabal cumplimiento de las condiciones pactadas y su conversión en resultados $^{8}$.

Asumida bajo estos parámetros, la reflexión sobre los procesos de dirección y gestión de las organizaciones públicas, parece acercarse cada vez más a las teorías tradicionales cuyos desarrollos han privilegiado enfoques y prácticas racionales y de cohesión organizacional. Desprovistos de instrumental adecuado para enfrentar y resolver la presencia de conflictos de poder, que resultan de las presiones por la ganancia, los expertos y empresarios han ido incorporando el manejo del conflicto como una variable más que se debe tener en cuenta en el comportamiento de las organizaciones. Utilizando desarrollos que van desde los modelos de la "tercera parte", que buscan la resolución de conflictos a través de la participación de un tercero (Brett y Golberg, 1983), hasta los modelos "integrativos que buscan resolver los conflictos a partir de la triada: motivación, control y liderazgo ${ }^{9}$, (Pruit, 1983) el manejo de conflictos organizacionales basados en la negociación ha venido ganando terreno en los círculos académicos y empresariales.

A diferencia de las organizaciones privadas, la consideración y manejo de conflictos en la gerencia de las interde- pendencias organizacionales, debe partir de tres supuestos básicos:

a. Las estructuras de poder en una organización pública, a pesar de tener su origen en la Constitución y las Leyes, son negociables, pero por referencia a una determinada correlación de fuerzas políticas generalmente externas a la organización;

b. Los contendientes, en el desarrollo de sus estrategias, pueden llegar a movilizar elementos de poder político o medios de presión social (ambos ajenos o no al control de la organización), en aras de preservar sus prerrogativas;

c. Las organizaciones públicas son sistemas dialécticos de negociación politica e institucional. (Bacharat, 1983)

En esta perspectiva, negociación y gestión de conflictos para la gerencia de las interrelaciones organizacionales, tienen lugar no solamente como un proceso de fuerzas centrífugas que busca operar sobre los conflictos posibles que puedan existir entre los objetivos e intereses propios de una organización determinada y los objetivos e intereses de las demás organizaciones sobre una operación específica. De lo que se trata, más bien, es de generar una dinámica relacional hacia afuera que le permita a la organización posicionarse con un producto y una mi-

8 En este sentido, la Gestión también se concibe como un proceso permanente de mediación (para la ruptura o concertación) de conflictos e intereses entre dos o más actores.

9 Utilizando la noción de "acuerdo integrativo" , por contraposición a la noción de "compromiso", Dean Pruitt en un excelente artículo expone cuatro razones por las cuales en una organización los negociadores de intereses buscan los acuerdos integrativos más que los compromisos, cuando a-. Las aspiraciones de las partes son muy altas, no hay solución si no se encuentra una forma de conciliar intereses; $b$. Un acuerdo integrativo tiene mayores probabilidades de ser estable; c-. Este tipo de negociación fortalece las relaciones entre las partes, de modo que puede atenuar posteriores conflictos; d-. Los acuerdos integrativos contribuyen al bienestar de una comunidad más amplia. 
sión institucional. También tienen lugar como un proceso de fuerzas centrípetas, en donde se procura integrar el conjunto de intereses y objetivos de los funcionarios con el conjunto de intereses y objetivos organizacionales que se tienen como entidad individual y como parte de un sistema. De lo que se trata, finalmente, es de desatar las dinámicas de acción institucional hacia adentro en función de un objeto y una misión definida.

\section{Elementos y enfoques para la gerencia en el sector público}

Los nuevos paradigmas de la Gerencia en el sector público enfatizan en tres aspectos básicos:

a. El proceso de toma de decisiones. Es el punto focal en donde se concentran en temas como los modos de formulación, implementación, seguimiento y evaluación de las políticas públicas esenciales, en los modelos de decisión en las alturas del poder o en la mejor administración de las empresas públicas por el gobierno central (Kliksberg).

b. Los sistemas de administración de personal. El reto consiste en pasar de sistemas administrativos en donde se manejan rutinas orientadas a la reafirmación de las jerarquías y el control fiscal, hacia un nuevo sistema en donde las políticas de personal basadas en la motivación, el rendimiento y la participación, se constituyen en el soporte principal de la organización;

c. Las estructuras administrativas. Partiendo de la convicción de que las estructuras perderán su primacía como componente rector de la capacidad organizacional, la cuestión del cambio se plantea en torno a la necesidad de integrar los procesos organizacionales en sistemas flexibles, en donde las organizaciones deben ser ambiguamente estructuradas, con el propósito explícito de erradicar aquellos problemas de jerarquías y vínculos de subordinación, que obstaculizan la mayor y más efectiva movilidad organizacional.

A pesar de que los nuevos paradigmas de la Gerencia reivindican una visión de integralidad y complejidad de las organizaciones, resulta evidente que las organizaciones públicas parecen estar más interesadas en acoger y adaptar las inno. vaciones empresariales producidas en el sector privado, que en producir sus propias innovaciones. El énfasis sobre los tres componentes principales de una organización (dirección, manejo de personal y estructura organizacional), no solamente dejan entrever una persistente visión autocentrada de las organizaciones en sí mismas y los empresarios en su ámbito de desempeño. También ponen en evidencia el propósito de adoptar el modelo más acabado de empresa privada, el más formalizado y competitivo en el mundo de los negocios que, tal como lo demuestra Crozier, resulta ser precisamente el que menos corresponde a los desarrollos y exigencias de la sociedad post-industrial.

\section{- Los enfoques convencionales}

En los últimos años, la administración moderna ha desarrollado novedosos conceptos y enfoques que, bajo el signo 
de la gerencia estratégica, buscan orientar el proceso mediante el cual una organización formula unos escenarios estratégicos y prospectivos, establece sus objetivos y define cómo llegar a ellos ${ }^{10}$. En la perspectiva de la "aplicación" de unos determinados conceptos y enfoques gerenciales, como paradigmas para la gerencia de las organizaciones públicas, se han invocado tres tipos de enfoques que, según sus promotores, por su contenido aportan muchos y muy novedosos elementos de organización y operación a los esfuerzos públicos por actuar de manera más eficiente.

El primer enfoque es el denominado de la administración por objetivos. A riesgo de reducir en exceso sus desarrollos, se puede decir que se trata de un modelo que, antes de buscar articular a la organización con las fuerzas del mercado, dirige todos sus esfuerzos al diseño de la ruta crítica que debe regir los procesos de toma de decisiones en una empresa privada de cualquier tamaño y escala. Desde la definición de la "misión"; su propósito central es el cómo definir los objetivos de la organización y evaluar sus resultados.

El segundo enfoque es el llamado de administración por políticas, que propone una manera distinta de operativizar la estructura organizacional y la función administrativa. Los principios básicos, parten de la gerencia estratégica en el sentido de que retoman la necesidad de definir claramente la misión de la organización, sus creencias y valores, sus estrategias y objetivos, sus políticas, así como las metas que se impone para crecer y orientarse hacia el futuro. La diferencia con la administración por objetivos, radica en el mayor énfasis que pone en el análisis del ciclo de control, el cual involucra acciones de "planear-hacer-verificar y actuar".

E tercer enfoque es el llamado control total de la calldad. Se trata, como afirma Cárdenas, de un enfoque gerencial estructurado para priorizar y resolver problemas, una filosofía empresarial que enfatiza en el desarrollo de una cultura de la calidad, unos principios sabios y orientadores del trabajo diario, y unas técnicas estadísticas y administrativas que apoyan este proceso en el que participan todos los miembros de la organización. (Cárdenas, 1990). Bajo el doble objetivo de crear una cultura de la calidad y de activar mecanismos que aseguren el desarrollo de la calidad como actitud organizacional, el enfoque del control total de calidad se fundamenta en una operación que comporta cuatro pasos: i. planear en función de las necesidades y expectativas del cliente, ii. implementar lo planeado; iii. verificar lo elecutado; y iv. actuar sobre lo verificado. En este enfoque, la utilización de los recursos está esencialmente concentrada en aquellas activida-

El carácter estratégico, proviene de la necesidad de que a través de unos determinados objetivos se puedan articular los requerimientos de competitividad y eficiencia que se pertilan de las exigencias y tendencias del mercado, con las estructuras y principios organizacionales generadas por un patrón de dirección administrativa y financiera de la organización. Un desarrollo más detallado se encuentra en Rosario del Río, "Los Enfoques Modernos de la Administración", Documento de Trabajo, 1992. 
des "estratégicas" que desarrollan los grados de satisfacción de los clientes.

Estos enfoques gerenciales tienen tres elementos en común:

a. Están concebidos para estructuras que son altamente descentralizadas. Es decir, para sistemas jerárquicos en donde las decisiones de la cúspide se delegan hasta los máximos niveles posibles y tales decisiones llegan y se ejecutan efectivamente en la base;

b. Suponen que los intereses de la organización, son siempre y en todos los casos, los intereses de sus empleados. No existe ningún tipo de conflicto entre los intereses personales de los trabajadores con los intereses institucionales;

c. Exigen que los niveles superiores de la organización tengan siempre, y en todos los casos, la autonomia para definir los objetivos de la organización y la discrecionalidad para controlar todas y cada una de las etapas y momentos de la operación organizacional.

\section{- Los nudos críticos de los enfo- ques convencionales}

En la perspectiva de los trazados de políticas públicas, los enfoques de administración por objetivos, administración por políticas y control total de la calidad encuentran dos grandes restricciones en su aplicación al sector público.

La primera, hace relación a cómo en las organizaciones públicas la descentralización (por delegación), de las decisiones y responsabilidades, se desarrolla no solamente en función de la existencia de unos determinados intereses y frentes de poder burocrático (que en todos los casos reflejan o dan lugar a nuevos nichos de poder efectivo). También tienen lugar en correspondencia con los montos de recursos humanos, técnicos y financieros disponibles, así como de los principios y parámetros de regulación administrativa y jurídica (régimen de contratación, de responsabilidades y competencias, administración y remuneración del personal, etc), que rigen el comportamiento de la organización.

La segunda restricción proviene del hecho de que la definición de objetivos en una organización pública se desarrolla, en la mayoría de los casos, como un proceso ajeno a los intereses y dinámica de la propia organización. (Azuero, 1990) Por una parte, está sujeto a unos principios jerárquicos y funcionales pre-determinados; son los niveles superiores los que, al optar por un determinado rumbo de gobierno, definen unos objetivos específicos a la acción del aparato estatal y a las organizaciones que lo componen. Por otra parte, su comportamiento está sometido a una determinada inercia del proceso presupuestal. Azuero muestra cómo los montos asignados a cada entidad y organización pública dependen, cuando se financian vía presupuesto, de los niveles ejecutados en el año anterior 0 , cuando se financian por sus propios recursos (via precios en empresas industriales y comerciales del Estado o tarifas en servicios públicos), dependen de los niveles de "subsidio" o "ayuda" (directa o indirecta, implícita o explicita) que defina el gobierno para la provisión de los bienes y servicios. Siguiendo con la argumentación de Azuero, las metas físicas que se 
establecen son por lo general el producto de las asignaciones presupuestales y no su premisa. El control presupuestal se entiende y aplica como garantía jurídica y administrativa sobre las operaciones, pero nunca se constituye en un parámetro adecuado para la eficiencia de la organización. (Azuero, 1990)

Sometidas a las restricciones polfticas y presupuestales, como a las presiones que ejercen las luchas por el poder burocrático, las organizaciones públicas para adquirir el carácter de "empresa pública" requieren, como condición, del desarrollo de una dinámica que estimule la iniciativa y promueva la innovación. Es decir, que aproveche y desarrolle el potencial que le ofrecen los recursos humanos, tecnológicos y financieros disponibles, en función de un propósito y en condiciones siempre cambiantes. Es el enfoque de la gerencia para la investigación, la capacitación y la gestión empresarial de los recursos disponibles. Investigación, para producir conocimiento que no sólo recupere e integre el saber-hacer social de los individuos a la organización, sino que permita el aprendizaje y la incorporación permanente de elementos de cambio; formación, para producir capacidad de los individuos internos y externos a la organización en actitud, aptitud y habilidad para el desarrollo de acciones siempre creadoras; gestión, para producir compromiso y conciencia social on torno a los aportes $y$ requerimientos que a la organización, como "empresa pública", le impone un determinado radio de acción.
- Elementos para un enfoque alternativo

La búsqueda de eficiencia, como parámetro del comportamiento empresarial de una organización pública, se proyecta en una doble perspectiva. Por una parte, desde el punto de vista de la gestión interna, el aprovechamiento empresarial de los recursos humanos, tecnológicos y financieros disponibles, exige dinámicas de dirección y operación basadas en la flexibilidad de las estructuras, la racionalización de los procedimientos. Por otra parte, en la perspectiva de las interrelaciones organizacionales, este enfoque gerencial implica que la eficiencla no está dada por la "correcta" e "intensiva" utilización de los recursos disponibles, sino por su grado de contribución al logro de los objetivos y metas definidas por un gobierno. Esto es, que lejos de ser considerada como el resultado de reducir los costos por unidad producida, la eficiencia se asume como la optimización del impacto de la inversión pública. Las autoridades de polftica no deben olvidar que la eficiencia no se mide por la intensidad de los factores involucrados en las acciones del Estado, sino por la magnitud de los cambios producidos sobre los parámetros de desarrollo que se quiere modificar.

Esta visión alternativa de eficiencia conduce al razonamiento propuesto por Crozier (1984), en el sentido de que to que venden las organizaciones públicas y estatales no es un producto o un servicio, sino calidad de vida de la población. Lo que venden es la posibilidad de vivir 
de otro modo. El consumidor, el usuario, es el propio y verdadero productor.

En este sentido, los enfoques gerenciales de la administración por objetivos, la administración por políticas y el control total de la calidad, en su sentido más tradicional, aparecen inútiles en su aplicación a las organizaciones públicas. Crozier argumenta que las viejas prácticas del "management" fordista que segmenta las acciones para luego estandarizarlas y hacerlas uniformes en un proceso administrativo y organizacional altamente concentrado, han perdido su vigencia. Lo que se requiere es considerar la capacidad y posibilidad de los recursos humanos para adaptarse al cambio y crecer aprovechando y desarrollando su inventiva y su potencial de contribución a los objetivos empresariales de la organización. En este contexto, adquiere sentido la consideración de que una empresa pública es competitiva sólo a condición que promueva la calificación de los recursos humanos, se informe sobre su localización estratégica y sobre las tendencias de los mercados (político y económico) y desarrolle una acción flexible y comprehensiva en su esfera de acción".

La Gerencia de las Interrelaciones Organizacionales consiste, entonces, en crear las condiciones institucionales indispensables para que no sólo las organizaciones del sector público operen de manera coordinada en función de unos propósitos de gobierno, sino también para que puedan formarse pequeñas comunidades sociales, alrededor de la prestación de un servicio o la producción de un bien público, sean capaces de establecer un intercambio intensivo de unas con otras y de éstas con el mundo real. No se trata de hacer planes o construir mercados, sino de suscitar interacciones que funcionen unas con otras. Como afirma Crozier, la verdadera revolución del management del cambio estructural, no es el de la apertura técnica, ni el producto, ni mucho menos los nuevos conceptos $y$ principios de la organización empresarial. La revolución está en la innovación social. Esto es, en la magnitud de los cam. bios que pueda producir (en términos de su capacidad de desatar procesos y formas particulares de organización social para un propósito especifico) sobre los parámetros de desarrollo que se quiera modificar.

Si hay algo que rescatar, de las nuevas formas de organización empresarial, es que la competitividad radica en el conocimiento. El enfoque de la gerencia para la investigación, la capacitación y la gestión de las interrelaciones organizacionales, exige del sector público una novedosa idea acerca de lo que significa invertir a futuro. Lo que se requiere, como argumenta Crozier:

\section{a. Invertir a futuro en el conoci-} miento, que permita no sólo recuperar el conjunto de experiencias e informaciones sociales acumuladas durante años por la "comunidad" y los funcionarios, sino también redescubrir las regulaciones e interferencias reales que afectan el comportamiento empresarial de la organización pública. Esto es, rescatar tanto los medios que permitan retomar el control sobre la organización e identificar nuevas formas de hacer las cosas, como los nuevos ejes potenciales que sean capaces 
de desatar y activar procesos sociales de cambio;

b. Invertir a futuro en el hombre, que permita no sólo desarrollar unas actitudes, aptitudes y capacidades para enfrentar y resolver problemas, sino que además genere compromiso social en la comunidad;

\section{c. Invertir a futuro en la organiza-} ción, que desate y active todas las formas posibles de experimentación en nuevas formas de organización, de acción institucional y de relación con su "clientela".

\section{Referencias Bibliográficas}

Azuero, Francisco. Requisitos para la Adopción de una Estrategia de Calidad y Productividad en el sector Público, en Gestión de Calidad y Productividad como estrategia de Desarrollo, Departamento Nacional de Planeación.

Bacharach, Samuel (1983). Bairgaining within organizations, on Max Bazerman and Roy Lewicky, Negotiating in Organizations, Editorial Beverly Hills, Sage Publications.

Bartos, Otomar (1974). Process and Outcome of Negotiation, Columbia Press University.

Brett, Jeanne y Stephen Golberg (1983). Mediator Avisers: a new third party role en Max Nazerman and Roy Lewicky, Negotiating in organizations, Editorial Beverly Hills, Sage Publications.

Brown, Michel y Steven Erie (1984). Poder y Administración: Paradigmas Alternativos para el análisis de la autonomia burocrática, en Oscar Oszlak, Teoria de la Burocracia Estatal: Enfoques Críticos, Editorial Paidos, Buenos Aires.

Cárdenas, Jorge Hernán. El Sector Público:
¿Comparable con el sector privado? Rev. Economla Colombiana, Contraloría General de la República.

Cárdenas, Jorge Hernán (1990). El Enfoque Gerencial del Control Total de Calidad en el Sector Público Colombiano, en Gestión de Calidad muy Productividad como estrategia de desarrollo. Memorias del Seminario Internacional, Cartagena, Junio 13 al 15.

Coase, R.H. (1996). La Naturaleza de la Empresa, saldrá en próximo número de la Rev. Cuadernos de Economia, Universidad Nacional de Colombia.

Crozier, Michel (1984). No se Cambia la Sociedad por Decreto, Instituto Nacional de Administración Pública, Colección Biblioteca Básica de Administración Pública, Madrid.

Crozier, Michel (1963). El Fenómeno Burocrático. Editions du Seuil.

Crozier, Michel. El Crecimiento del Aparato Administrativo en el Mundo de la Complejidad, Obligaciones y Oportunidades, del estado Arrogante al estado moderno.

Crozier, Michel. El Crecimiento del Aparato Administrativo en el Mundo de la Complejidad. Obligaciones y oportunidades. Del estado Arrogante al estado Modesto.

Faulkner, Hugh (1992) Un Vistazo a la Gerencia Pública, tomado de "The Journal of the Institute of Public Administration, Fall/Automne, Toronto 1990, págs. 383-388. Traducción de Hernando Loaiza ESAP.

Kennedy, Gavin y John Mcmillan (1985). Cómo Negociar con Exito, Editorial Deusto, Bilbao.

Huntington, Samuel (1991). El Orden Politico en las Sociedades en Cambio. $2^{*}$ reimpresión, Editorial Paidos, Buenos Aires.

Israel, Arturo. Reformas a la Función del estado. 
Kliksberg, Bernardo (1984). La reforma Administrativa en América Latina, en Teoria de la Burocracia Estatal: Enfoques Críticos, Oscar Oszlak (comp.), Editorial Paidos, Buenos Aires

Klisberg, Bernardo (1990). Nuevas Fronteras Tecnológicas en la Gerencia Pública. Buenos Aires.

Lorino Philippe (1991). El Control de Gestión Estratégica, Editorial Dunod, París.

Majone, Giandomenico (1992). La Factibilidad de las Politicas Sociales, en Luis F. Aguilar Villanueva, La Hechura de las Politicas, Grupo Editorial Miguel Angel Porrua, México.

Márquez, Viviane y Rainer Godan (1992). Burocracia y Políticas Públicas: Perspectivas desde América Latina, México.

Medelín Torres, Pedro (1992a). Elementos para la Creación y Puesta en Marcha de una Escuela de Gobiemo Territorial. Documento de Trabajo Corpes, Diciembre 1992.

Medelin Torres, Pedro (1992b). ¿Democracia Inacabada, transición o Ficción Democrática? Los Límites entre la reestructuración y el reformismo en Amé- rica Latina. Nuevos itinerarios para un viejo problema. Documento de trabajo, CIDER.

Medellín Torres, Pedro (1992c). Gerencia Pública en la Perspectiva de la Modernización y la Apertura, Rev. De la Es. cuela Superior de Administración Pública, Santafé de Bogotá.

Medellin Torres, Pedro (1993). La gestión de Gobierno: Política y Administración, presentado al Seminario Nacional Gestión Política para Políticos, $\mathrm{Cl}$ DER-EAFIT, Medellín.

Oszlak, Oscar (1989). Regímenes Políticos y políticas Públicas. Reflexiones a partir de algunas experiencias latinoamericanas. Buenos Aires.

Pruitt, Dean G. (1983). Achieving Integrative Agreements, en Max Bazerman and Roy Lewicky, Negotiating in Organizations, Editorial Beverly Hills, Sage Publications.

Río, Rosario del (1992). Los Enfoques Modernos de la Administración. Documento de trabajo.

Shelling, Thomas (1964). La estrategia del Conflicto, Editorial Tecnos, Madrid. 